\title{
Medicine of the Future: Pessimistic Versus Optimistic Prognosis
}

Olga Golubnitschaja*

Department of Radiology, Rheinische Friedrich-Wilhelms-University of Bonn, Germany

\begin{abstract}
Pessimistic versus optimistic prognosis is considered for the healthcare systems worldwide depending on the paradigm which medical survices will apply over the next 2 decades. Current healthcare deals with dramatic problems created by the concept of delayed (or reactive) medical approaches resulting in a low cost effectiveness of treatments and outcomes considered as inadequate for many acute and chronic pathologies. In contrast, Predictive Preventive and Personalised Medicine (PPPM) is the new philosophy utilising an integrative concept of medical services that enables to predict individual predisposition before onset of the disease, to provide targeted preventive measures and create personalised treatment algorithms tailored to the person. The optimistic outcomes by PPPM are well justified as promoting healthcare sector by following measures: effective prevention early in life, identification of persons at-risk, patients' stratification, optimal therapy planning, prediction and reduction of adverse drug-drug or drug-disease interactions, etc. Emerging technologies, such as omics, medical imaging, pharmacogenetics, pathology-specific patterns of biomarkers, disease modelling, individual patient profiles, etc. are expected to play a pivotal role in the advancing of medical services. Being at the forefront of this process, the European Association for Predictive, Preventive and Personalised Medicine promotes the integrative concept of PPPM among professionals, governmental institutions, funding bodies, patient organisations and in the public domain. An integrative approach by PPPM is considered as the medicine of the future.
\end{abstract}

Keywords: Predictive Preventive Personalised Medicine (PPPM); Healthcare; Omics; Medical imaging; Education; Diabetes; Cancer; Neurodegenerative diseases; Innovative strategy; Advanced technologies; European Association for Predictive, Preventive and Personalised Medicine (EPMA)

\section{Current Healthcare: Challenges and Considerations}

Current healthcare outcomes are considered as being inadequate for many acute and chronic disorders. As the matter of fact, severe chronic pathologies are treated after onset of the disease, frequently at near endstages. This considers a spectrum of cardiovascular disorders, type 2 diabetes mellitus, common neurodegenerative pathologies and several cancer types. Global figures cry for a necessity in predictive diagnosis, targeted preventive measures and treatments tailored to the person. Following facts about the current diabetes care argue for themselves:

- Worldwide, every 10 seconds one patient dies from DM and DMrelated complications.

- The worldwide estimated impact of undiagnosed DM and DMrelated complications ranges between 30 and 50\% for DM type 2 .

- A dynamic increase in DM-affected subpopulations is well documented: the epidemic scale, year-by-year it is getting more pessimistic as demonstrated in figure 1 [1].

- DM type 2, historically characterized as an adult-onset disorder, now comprises approximately $30 \%$ of new DM-cases already in the second decade of life, i.e. among teenagers.

- Early onset of DM type 2 leads to a dramatic shift in the oveall appearance of complications characteristic for DM-patients, namely early and highly increased risk as well as poor prognosis for cardiovascular, neurodegenerative and cancer diseases developted secondarily to DM. Direct consequences include: significantly shorter life-span expectation, marked lower life-quality and dramatic increase in the cost burden per DM-patient multiplied by the currently high and epidemically increasing part of the DM-affected subpopulation in the society.

A pandemic scenario for type 2 diabetes mellitus, common neurodegenerative disorders and some types of cancer over the next 10-20 years is considered by a pessimistic prognosis followed by the economic disaster of healthcare systems in a global scale.

\section{Advanced Healthcare Tailored to the Person: What is beyond the Issue?}

The principle difference between the current and highly-desirable healthcare is the progress from the unsatisfactory delayed reactive to the advanced predictive and preventive medical health services as summarised in figure $2[2]$.

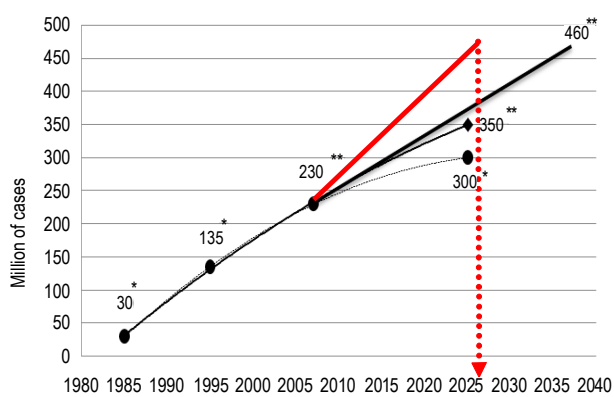

Year

*Estimations as published in 2000; ${ }^{* *}$ worsening prognosis as published in 2003-2012; the youngest prognosis is marked in red colour [1].

Figure 1: Worldwide prognosis of the epidemic scale in diabetes.

*Corresponding author: Olga Golubnitschaja, Department of Radiology Rheinische Friedrich-Wilhelms-University of Bonn, Germany, E-mail: Olga.Golubnitschaja@ukb.uni-bonn.de

Received December 06, 2012; Accepted December 14, 2013; Published December 16, 2013

Citation: Golubnitschaja O (2013) Medicine of the Future: Pessimistic Versus Optimistic Prognosis. Altern Integ Med 1:e105. doi:10.4172/2327-5162.1000e105

Copyright: @ 2013 Golubnitschaja O. This is an open-access article distributed under the terms of the Creative Commons Attribution License, which permits unrestricted use, distribution, and reproduction in any medium, provided the original author and source are credited. 
Citation: Golubnitschaja O (2013) Medicine of the Future: Pessimistic Versus Optimistic Prognosis. Altern Integ Med 2:e105. doi:10.4172/23275162.1000 e105

Being at the forefront of this process, the European Association for Predictive, Preventive and Personalised Medicine (EPMA, www. epmanet.eu) promotes the paradigm change from delayed interventional to predictive medicine tailored to the person, from reactive to preventive medicine and from disease to wellness. The integrative concept of PPPM is emerging as the focal point of efforts in healthcare aimed at curbing the prevalence of both communicable and non-communicable diseases. The critical role of PPPM in modernisation of healthcare has been acknowledged by the global strategic organizations such as UNO and the National Institutes of Health. However, the "success story" of

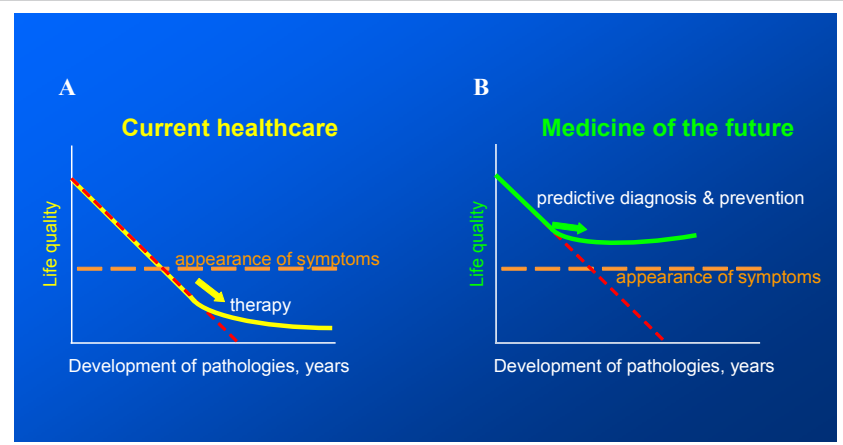

Figure 2: Schematic presentation of the principle difference between the currently applied reactive healthcare $(A)$ and the highly-desirable predictive diagnostics followed by targeted preventive measures and treatments tailored to the person $(B)[2]$ the extensive realisation of PPPM in medical services depends very much on the consistent implementation of innovative approaches such as the predictive and prognostic omics technologies completed by the cost-effective management of diseases.

\section{References}

1. Golubnitschaja O (2012) Advances in Predictive, Preventive and Personalized Medicine. Springer, New York, USA

2. Golubnitschaja O (2009) Predictive diagnostics and personalized treatment Dream or Reality. Nova Science Publishers, New York, USA.

Submit your next manuscript and get advantages of OMICS Group submissions

Unique features:

- User friendly/feasible website-translation of your paper to 50 world's leading languages

Audio Version of published paper

Digital articles to share and explore

Special features:

250 Open Access Journals

20,000 editorial team

21 days rapid review process

Quality and quick editorial, review and publication processing

Indexing at PubMed (partial), Scopus, EBSCO, Index Copernicus and Google Scholar etc

Sharing Option: Social Networking Enabled

- Authors, Reviewers and Editors rewarded with online Scientific Credits

Better discount for your subsequent articles

Submit your manuscript at: http://www.omicsonline.org/submission 